\title{
Complexity Analysis on Normal and Abnormal Electrogastrograms Using Tsallis Entropy
}

\author{
Paramasivam Alagumariappan *, Arivarasu Rajagopal and Kamalanand Krishnamurthy \\ Department of Instrumentation Engineering, MIT Campus, Anna University, Chennai 600025, India \\ *Correspondence: parama.ice@gmail.com; Tel.: +91-950-062-0901
}

\begin{abstract}
Electrogastrograms (EGG) are electrical signals generated by the muscles of the stomach and the features of these signals can be used to diagnose several digestive disorders. Entropy is a measure of the disorder associated with a system and hence is a measure of complexity of the system. In medical diagnostics, entropy has proved to be an efficient feature for discriminating the normal and abnormal states of biological systems. In this work the EGG signals have been obtained from normal and abnormal subjects having different digestive abnormalities (diarrhea, vomiting and stomach ulcer), from a local hospital. Further, the Tsallis entropy associated with the collected signals has been analyzed. Results demonstrate that the Mean Tsallis Entropy (MTE) (with $\alpha=0.5$ ) of the EGG signals obtained from normal individuals (MTE=313.861) is high when compared to the individuals having diarrhea (MTE=278.0259), vomiting (MTE=105.1278) and stomach ulcer (MTE=-839.201). Since, entropy is the complexity associated with the signal, it is found that the complexity of the normal EGG signals is high when compared to the abnormal EGG signals. This work appears to be of high clinical relevance, since feature extraction from EGG signals is highly useful for diagnosis of digestive abnormalities.
\end{abstract}

Keywords: entropy; Tsallis entropy; electrogastrograms; digestive disorders

\section{Introduction}

The gastrointestinal tract (GI) or digestive tract is made up of the esophagus, stomach, large and small intestines, liver, pancreas and the gallbladder. The process of digestion has several stages in which the food is broken into smaller parts and the nutrients are absorbed [1]. Digestive diseases are disorders of the digestive tract. The initial stage of problems in the digestive tract includes several symptoms like bleeding, bloating, diarrhea, constipation, heartburn, nausea, vomiting etc. Investigation of digestive disorders includes upper GI endoscopy, ultrasound endoscopy, Electrogastrography etc. In most cases, the diagnosis of the digestive diseases is invasive and a complex task and Electrogastrography (EGG) can serve as an efficient and noninvasive alternative for diagnosis of digestive disorders [2].

For measurement of EGG signals, several electrodes are placed onto the abdomen over the stomach. The electrodes sense the electrical signals originating from the stomach muscles. Some of the features of the EGG signals of normal individuals differ when compared to the features of the signals obtained from patients with abnormalities [1]. By analyzing such features, several digestive disorders can be diagnosed. The entropy associated with a signal is a measure of the information content of the signal and is a method for quantifying the disorder associated with the system that generates the signal. In recent years, biomedical researchers have adopted various entropy measures as features for analysis of complex biosignals [3-7].

Gopu et al. (2008) [2] have acquired and analyzed the Electrogastrograms for Digestive System Disorders such as Dyspepsia, Stomach Ulcer, Nausea etc. Further, the authors have discussed the dissimilarity in frequency and amplitude of Electrogastrograms. Riezzo et al. (2013) [8] have discussed the recording and processing methodology of the Electrogastrograms. Further, the 
The 3rd International Electronic and Flipped Conference on Entropy and Its Applications (ECEA 2016), 1-10 November 2016; Sciforum Electronic Conference Series, Vol. 3, 2016

authors have presented clinical applications of Electrogastrograms such as detection of digestive abnormalities in adults and children. Al-nuaimi et al. (2015) [9] have proposed the most promising information theoretic methods for quantifying changes in the EEG using Tsallis entropy. De Bock et al. (2010) [10] have utilized the Tsallis entropy of EEG signals for early detection of Alzheimer's disease. Further, the authors have concluded Tsallis entropy based EEG analysis was a highly promising potential diagnostic tool for mild cognitive impairment and early dementia.

The objective of this work is to analyze normal and abnormal Electrogastrograms in cases of various digestive disorders such as diarrhea, vomiting and stomach ulcer, using Tsallis and Renyi entropy.

\section{Materials and Methods}

\subsection{Design of EGG Acquisition System}

Figure 1 shows the developed circuit for acquiring EGG signals using instrumentation amplifiers.In this EGG measurement system, two cutaneous electrodes are used (one as reference electrode and the other as measurement electrode). The electrodes are placed on the stomach, according to the measurement protocol [11], with a distance of $7 \mathrm{~cm}$ between the electrodes.

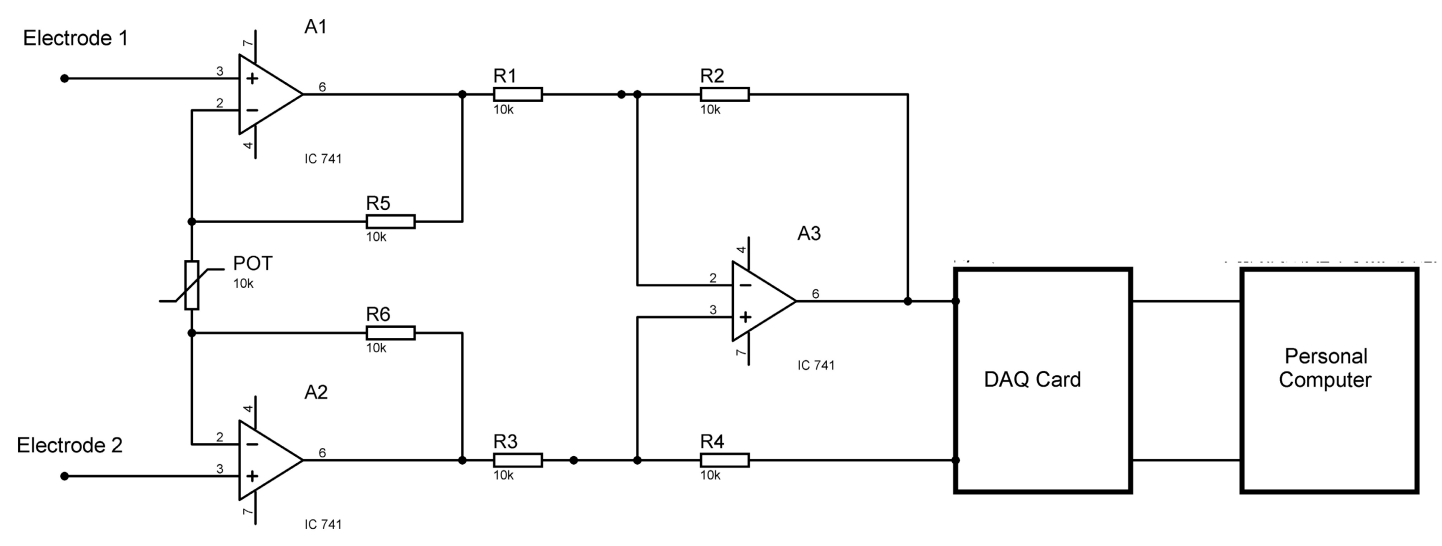

Figure 1. The developed circuit for EGG acquisition system.

The EGG signals were acquired and logged using the data acquisition system coupled with LabVIEW (V14.0.1).The EGG signals from normal and abnormal human subjects were acquired under the guidance of a medical Personnel at a local hospital.

\subsection{Analysis of Digestive Disorders from Recorded EGG Signals Using Tsallis and Renyi Entropy}

Entropy is a measure of the disorder associated with a system and hence is a measure of complexity of the system [12]. In medical diagnostics, entropy has proved to be an efficient feature for discriminating the normal and abnormal states of biological systems.There are several entropic measures such as Tsallis entropy, Renyi entropy, Shannon's entropy, Approximate entropy, fuzzy entropy etc. In this work, the Tsallis and Renyi entropy measures have been utilized for analysis of the acquired EGG signals. Tsallis entropy $\left(H_{\mathrm{R}}\right)$ is defined as [13]:

$$
H_{R}=\frac{1}{\alpha-1}\left(1-\sum_{i=1}^{n} p_{i}^{\alpha}\right)
$$

where, $P_{i}$ is a given set of probabilities and $\alpha$ is a real number. Increasing the value of $\alpha$ results in more contribution of high probabilities than low probabilities for the entropy values [13]. Renyi entropy $(H(a))$ is defined as [12]: 
The 3rd International Electronic and Flipped Conference on Entropy and Its Applications (ECEA 2016), 1-10 November 2016; Sciforum Electronic Conference Series, Vol. 3, 2016

$$
H(a)=\frac{1}{1-a} \log _{2}\left(\sum_{i=1}^{n} p_{i}^{a}\right)
$$

where, $P_{i}$ is the probability that a random variable takes a given value out of $n$ values, and $\alpha$ is the order of the entropy measure. As $\alpha$ increases, the entropy values become more sensitive to higher probabilities and less sensitive to lower probabilities [12]. Tsallis entropy is one of the most promising information theoretic methods for biosignal analysis [9] and Renyi entropy is an effective measure of complexity of the signal [15]. The Mean Tsallis Entropy (MTE) and Mean Renyi Entropy (MRE) at different $\alpha$ values were calculated for the acquired EGG signals from the normal and abnormal cases. Further, the effects of the digestive disorders on the entropy values of EGG signals were analyzed.

\section{Results and Discussion}

Figure 2 shows the Typical EGG signals recorded for normal and abnormal individuals with various digestive disorders such as diarrhea, vomiting and stomach ulcer. Several variations in the EGG signals are observed in normal and abnormal cases.

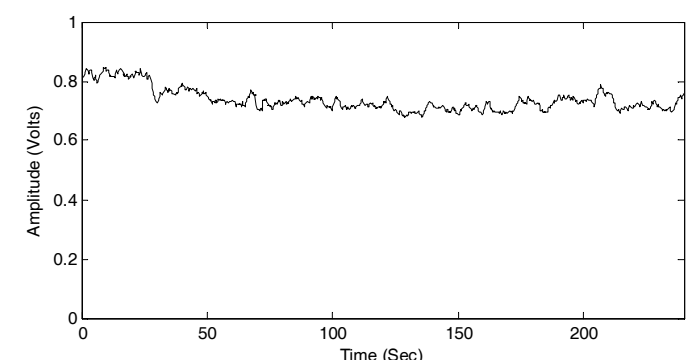

(a)

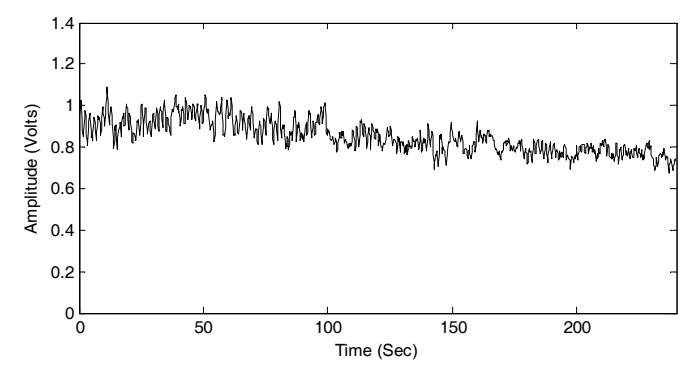

(c)

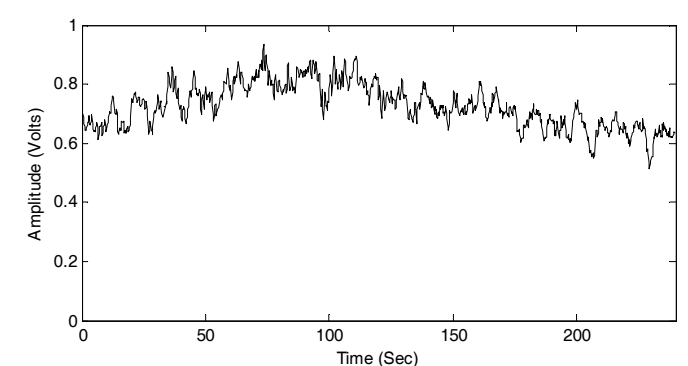

(b)

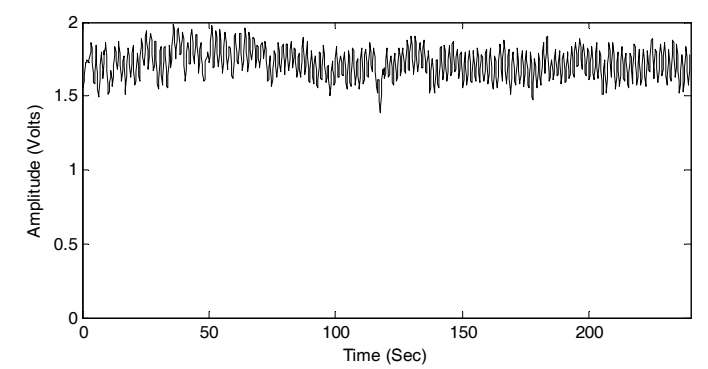

(d)

Figure 2. Typical EGG signal recorded from (a) normal and abnormal individuals suffering from (b) diarrhea, (c) vomiting and (d) stomach ulcer.

Figure 3 shows the mean Tsallis entropy and mean Renyi entropy of EGG signals acquired from normal and abnormal individuals, as a function of order $\alpha$. It is seen that the mean Tsallis entropy decreases with increase in $\alpha$. Further, it is found that the mean Tsallis entropy values of normal individual is higher when compared to the entropy values of individuals having abnormalities, at different $\alpha$ values. It is seen that the mean Renyi entropy increases with increase in $\alpha$. Further, it is found that the mean Renyi entropy values of normal individual is low compared to the Renyi entropy values of individuals having abnormalities for different $\alpha$ values. 
The 3rd International Electronic and Flipped Conference on Entropy and Its Applications (ECEA 2016), 1-10 November 2016; Sciforum Electronic Conference Series, Vol. 3, 2016

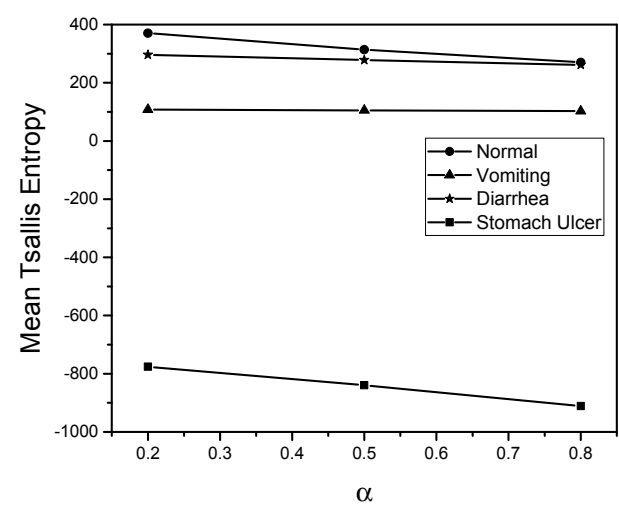

(a)

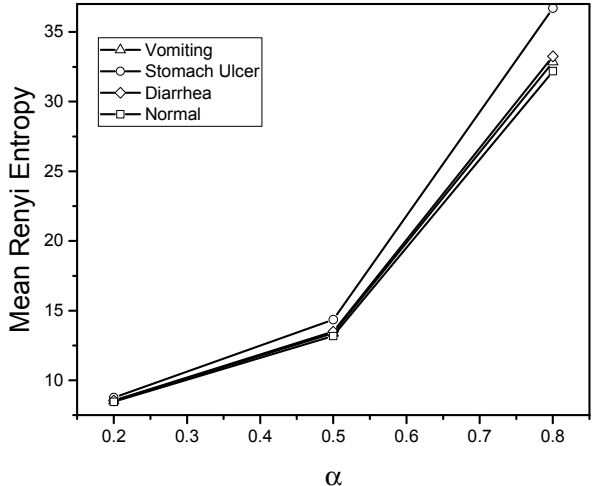

(b)

Figure 3. Mean entropy value (a) Mean Tsallis Entropy (MTE) and (b) Mean Renyi Entropy (MRE) of normal and abnormal EGG signals, shown as a function of $\alpha$.

The Normalized Mean Tsallis Entropy (NMTE) and the Normalized Mean Renyi Entropy (NMRE) values at three different orders $(\alpha=0.2, \alpha=0.5$ and $\alpha=0.8)$ for normal and abnormal individuals (diarrhea, vomiting and stomach ulcer) are presented in Table 1. The normalization was performed using Min-Max normalization approach. It is seen that, in all the cases, the normalized mean Tsallis entropy values for normal individual is higher when compared to the entropy values for abnormal individuals with various digestive disorders such as diarrhea, vomiting and stomach ulcer, at three different orders ( $\alpha=0.2, \alpha=0.5$ and $\alpha=0.8)$. Further, it is observed that the Tsallis entropy value for normal individuals is highest at $\alpha=0.2$ and the entropy value for individuals having stomach ulcer is lowest at $\alpha=0.8$.

It is found that, in all the cases, the normalized mean Renyi entropy values for normal individual is low when compared to the normalized mean Renyi entropy values for abnormal individuals with various digestive disorders such as diarrhea, vomiting and stomach ulcer at three different orders ( $\alpha=0.2, \alpha=0.5$ and $\alpha=0.8$ ). Further, it is observed that the Renyi entropy value for normal individuals is lowest at $\alpha=0.2$ and the Renyi entropy values for individuals having stomach ulcer is highest at $\alpha=0.8$.

Table 1. Normalized Mean Tsallis Entropy (NMTE) and Normalized Mean Renyi Entropy (NMRE) values for EGG signals acquired from normal and abnormal subjects.

\begin{tabular}{|c|c|c|c|c|c|c|}
\hline \multirow[t]{2}{*}{ Subjects } & \multicolumn{3}{|c|}{ Normalized Mean Tsallis Entropy (NMTE) } & \multicolumn{3}{|c|}{$\begin{array}{c}\text { Normalized Mean Renyi Entropy } \\
\text { (NMRE) }\end{array}$} \\
\hline & $\alpha=0.2$ & $\alpha=0.5$ & $\alpha=0.8$ & $\alpha=0.2$ & $\alpha=0.5$ & $\alpha=0.8$ \\
\hline normal & 1 & 1 & 1 & 0 & 0 & 0 \\
\hline diarrhea & 0.9352 & 0.9689 & 0.9927 & 0.9866 & 0.9664 & 0.9464 \\
\hline vomiting & 0.7711 & 0.8199 & 0.8578 & 0.9921 & 0.9757 & 0.9611 \\
\hline $\begin{array}{l}\text { stomach } \\
\text { ulcer }\end{array}$ & 0 & 0 & 0 & 1 & 1 & 1 \\
\hline
\end{tabular}

\section{Conclusions}

Electrogastrography is a noninvasive technique to record the electrical activity of the digestive system and the features obtained from these signals are useful for diagnosis and staging of several digestive diseases. Entropy is a measure of the disorder associated with a system and hence is a measure of the information content, uncertainty and complexity of the system. In medical 
The 3rd International Electronic and Flipped Conference on Entropy and Its Applications (ECEA 2016), 1-10 November 2016; Sciforum Electronic Conference Series, Vol. 3, 2016

diagnostics, entropy has been used as an efficient feature for classification of normal and abnormal states of physiological systems. In this work the EGG signals have been obtained from normal and abnormal subjects having different digestive abnormalities (diarrhea, vomiting and stomach ulcer), from a local hospital. Further, the Tsallis entropy and Renyi entropy of the acquired signals have been estimated and the entropy of normal and abnormal EGG signals is analyzed. Results demonstrate that the Mean Tsallis Entropy of the EGG signals obtained from normal individuals is high when compared to the individuals having diarrhea, vomiting and stomach ulcer. This work appears to be of high clinical relevance, since feature extraction from EGG signals is highly useful for noninvasive diagnosis of various digestive abnormalities.

Acknowledgements: The authors thank Sree Balaji Medical College and Hospital, Chennai, Tamil Nadu, India for helping towards acquiring the EGG signals for carrying out this research work.

Author Contributions: Paramasivam Alagumariappan and Kamalanand Krishnamurthy conceived the idea of this manuscript. Arivarasu Rajagopal designed the experiments and acquired the signals. Paramasivam Alagumariappan and Kamalanand Krishnamurthy have performed the analysis. All the authors have contributed towards preparing the manuscript. All authors have read and approved the final manuscript.

Conflicts of Interest: The authors declare no conflict of interest.

\section{References}

1. Gopu, G.; Neelaveni, R.; Porkumaran, K. Acquisition and analysis of electrogastrogram for digestive system disorders using a novel approach. In Proceedings of International Conference on Electrical and Computer Engineering 2008 (ICECE 2008), Dhaka, Bangladesh, 20-22 December 2008; pp. 65-69.

2. Gopu, G.; Neelaveni, R.; Porkumaran, K. Investigation of digestive system disorders using Electrogastrogram. In Proceedings of International Conference on Electrical and Computer Engineering 2008 (ICECE 2008), Dhaka, Bangladesh, 20-22 December 2008; pp. 201-205.

3. Li, Y.; Fan, X.; Li, G. Image segmentation based on Tsallis-entropy and Renyi-entropy and their comparison. In Proceedings of the 4th IEEE International Conference on Industrial Informatics, Singapore, 16-18 August 2006; pp. 943-948.

4. Lima, C.F.L.; Assis, F.M.; de Souza, C.P. A comparative study of use of Shannon, Rényi and Tsallis entropy for attribute selecting in network intrusion detection. In Proceedings of 2011 IEEE International Workshop on Measurements and Networking (M\&N), Anacapri, Italy, 10-11 October 2011; pp. 77-82.

5. Xu, Q.; Sbert, M.; Xing, L.; Zhang, J. A novel adaptive sampling by tsallis entropy. In Proceedings of International Conference on Computer Graphics, Imaging and Visualisation 2007 (CGIV'07), Sydney, Australia, 14-17 August 2007; pp. 5-10.

6. Kaufman, M.; Zurcher, U.; Sung, P.S. Entropy of electromyography time series. Physica A 2007, 386, 698-707.

7. Maszczyk, T.; Duch, W. Comparison of Shannon, Renyi and Tsallis entropy used in decision trees. In Artificial Intelligence and Soft Computing-ICAISC 2008; Springer: Berlin/Heidelberg, Germany, 2008; pp. 643-651.

8. Riezzo, G.; Russo, F.; Indrio, F. Electrogastrography in adults and children: the strength, pitfalls, and clinical significance of the cutaneous recording of the gastric electrical activity. BioMed Res. Int. 2013, doi: $10.1155 / 2013 / 282757$.

9. Al-nuaimi, Ali H.; Jammeh, E.; Sun, L.; Ifeachor, E. Tsallis entropy as a biomarker for detection of Alzheimer's disease. In Proceedings of the 37th Annual International Conference of the IEEE Engineering in Medicine and Biology Society (EMBC), Milano, Italy, 25-29 August 2015; pp. 4166-4169.

10. De Bock, T.J.; Das, S.; Mohsin, M.; Munro, N.B.; Hively, L.M.; Jiang, Y.; Smith, C.D.; Wekstein, D.R.; Jicha, G.A.; Lawson, A. et al. Early detection of Alzheimer's disease using nonlinear analysis of EEG via Tsallis entropy. In Proceedings of Biomedical Sciences and Engineering Conference (BSEC) 2010, Oak Ridge, TN, USA, 25-26 May 2010; pp. 1-4. 
The 3rd International Electronic and Flipped Conference on Entropy and Its Applications (ECEA 2016), 1-10 November 2016; Sciforum Electronic Conference Series, Vol. 3, 2016

11. Parkman, H.P.; Hasler, W.L.; Barnett, J.L.; Eaker, E.Y. Electrogastrography: a document prepared by the gastric section of the American Motility Society Clinical GI Motility Testing Task Force. Neurogastroenterol. Motil. 2003, 15, 89-102.

12. Cornforth, D.J.; Tarvainen, M.P.; Jelinek, H.F. How to calculate Renyi entropy from heart rate variability, and why it matters for detecting cardiac autonomic neuropathy. Front. Bioeng. Biotechnol. 2014, doi: 10.3389/fbioe.2014.00034.

13. Gajowniczek, K.; Ząbkowski, T.; Orłowski, A. Comparison of Decision Trees with Rényi and Tsallis Entropy Applied for Imbalanced Churn Dataset. Ann. Comput. Sci. Inf. Syst. 2015, 5, 39-44.

14. Ravariu, C.; Ursutiu, D.; Babarada, F.; Arhip, J.; Arama, S.S.; Radulian, G.; Samoila, C. Remote measurements of the electrical gastric signals-between theory and practice. In Proceedings of the 11th International Conference on Remote Engineering and Virtual Instrumentation (REV), Porto, Portugal, 26-28 February 2014; pp. 281-284.

15. Gonzalez Andino, S. L.; Grave de Peralta Menendez, R.; Thut, G.; Spinelli, L.; Blanke, O.; Michel, C. M.; Landis, T. Measuring the complexity of time series: an application to neurophysiological signals. Hum. Brain Mapp. 2000; 11, 46-57.

16. Kasicka-Jonderko, A.; Jonderko, K.; Krusiec-Swidergol, B.; Obrok, I.; Blonska-Fajfrowska, B. Comparison of multichannel electrogastrograms obtained with the use of three different electrode types. J. Smooth Muscle Res. 2006, 42, 89-101.

17. Yin, J.; Chen, J.D.Z. Electrogastrography: methodology, validation and applications. J. Neurogastroenterol. Motil. 2013, 19, 5-17.

18. Cornforth, D.J.; Tarvainen, M.P.; Jelinek, H.F. Using renyi entropy to detect early cardiac autonomic neuropathy. In Proceedings of the 35th Annual International Conference of the IEEE Engineering in Medicine and Biology Society (EMBC), Osaka, Japan, 3-7 July 2013; pp. 5562-5565.

19. Chen, J.D.Z. Non-invasive measurement of gastric myoelectrical activity and its analysis and applications. In Proceedings of the 20th Annual International Conference of the IEEE Engineering in Medicine and Biology Society, Hong Kong, China, 29 October-1 November 1998; Volume 6, pp. 2802-2807.

20. Patterson, M.; Rintala, R.; Lloyd, D.; Abernethy, L.; Houghton, D.; Williams, J. Validation of electrode placement in neonatal electrogastrography. Dig. Dis. Sci. 2001, 46, 2245-2249.

21. Brown, B.H.; Smallwood, R.H.; Duthie, H.L.; Stoddard, C.J.; Intestinal smooth muscle electrical potentials recorded from surface electrodes. Med. Boil. Eng. 1975, 13, 97-103.

22. Bromiley, P.A.; Thacker, N.A.; Bouhova-Thacker, E. Shannon entropy, Renyi entropy, and information. Available online: http://www.tina-vision.net/docs/memos/2004-004.pdf (accessed on 21 October 2016).

(C) 2016 by the authors. Submitted for possible open access publication under the terms and conditions of the Creative Commons Attribution (CC-BY) license (http://creativecommons.org/licenses/by/4.0/). 